\title{
Performance Comparison of Sphere Decoding and Global Search Algorithm based Multiuser Detectors for MC-CDMA System under Clipping Noise
}

\author{
S.Sivanesskumar \\ Assistant Professor, Department of IT \\ Anjalai Ammal Mahalingam Engineering College, \\ Kovilvenni, Tamilnadu, India.
}

\author{
Dr R.Sukanesh \\ Professor, Department ECE \\ Thiagarajar College of Engineering, \\ Madurai, Tamilnadu, India
}

\begin{abstract}
Multi Carrier Code Division Multiple Access (MC-CDMA), a promising technology for the $4 \mathrm{G}$ communication systems is considered in this paper. The foremost limitation of such system is the Multiple Access Interference (MAI) which is due to frequency-selective fading, near-far effect, frequency offset, and nonlinear power amplification due to clipping noise. The performance of MC-CDMA under such scenario is poor and optimal detection is one of the solutions with a high complexity is required. In this paper the Bit Error Rate (BER) performance is compared under clipping noise with sphere decoding and Global search algorithm based Multiuser detectors.
\end{abstract}

Key Words: MC-CDMA System, Multiuser detectors, Clipping Noise, Sphere decoding, Global search algorithm.

\section{INTRODUCTION}

CDMA achieves soft capacity limit by using the same bandwidth all time by assigning different spreading codes to each user. The only limitation is the self interference and multiuser interference [1,2]. One of the reasons for this impairment is contributed by the channel. Orthogonal Frequency Division Multiple Access (OFDM) is another technology adopted by the digital broadcasting society to counter the Inter Symbol Interference (ISI) problem. The fusion of OFDM and CDMA has yielded MC-CDMA which applies the spreading sequences in the frequency domain. MC-CDMA uses spreading in frequency domain. It is opted for the downlink where the spectral efficiency is important and the high data rate is required $[2,3,4]$. The MC-CDMA in presence of non linear distortion and Multiple Access Interference (MAI) is dealt with various detectors $[5,6]$ using Sphere decoding and Genetic Algorithms [7]. Other global optimization technique like Global Search (GS) which can be implemented in a parallel processor is discussed in this paper.

This paper is organized as follows. Section 2 describes the system description; Section 3 discusses the optimum receiver; Section 4 describes clipping noise effects;

Section 5 describes the Sphere decoding; Section 6 describes the Global Search algorithm based optimum detector; Section 7 gives the simulation results and Section 8 draws the conclusion.

\section{SYSTEM DESCRIPTION}

In the transmitter for the MC-CDMA, data from $\mathrm{k}$ users are spread using different spreading sequences of length $L$ and they are combined. This is explained in the following equations [3].
$U_{k}$ is the $k^{\text {th }}$ user with data rate $1 / T_{d}$. The spreading code is $C k_{0}, C k_{2}, \ldots C k_{L-1}$. After spreading of each user and combining, the signal is $S k_{0}, S k_{1}, \ldots S k_{L-1}$

$S=\sum_{k=0}^{k-1} s_{k}=\left(s_{0}, s_{1}, \ldots \ldots, s_{L-1}\right)^{T}$

Where,

$$
\begin{aligned}
& S=C U \\
& U=\left(U_{0}, U_{1}, \ldots . . U_{k-1}\right)^{T} \\
& C=\left(C_{0}, C_{1}, \ldots . . C_{k-1}\right)^{T}
\end{aligned}
$$

The combined signal is send to serial to parallel converter and each data element is given a particular frequency slot using IFFT. Then the output is combined to form a OFDM symbol.

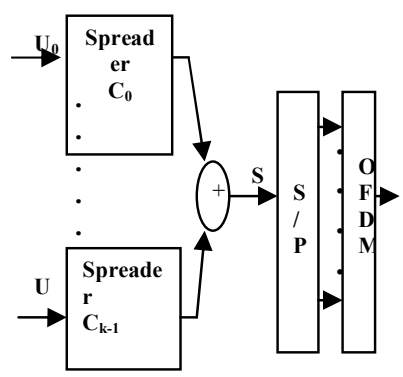

Figure 1. Transmitter configuration of a MC-CDMA system

Now the received OFDM symbol will be given as

$$
\begin{aligned}
& r=\sum_{k=0}^{k-1} H_{k} S_{k}+\eta=\left(R_{0}, R_{1}, \ldots . . R_{L-1}\right) \\
& r=H S+\eta
\end{aligned}
$$

The " $\mathbf{r}$ " is the received OFDM symbol but a signal element from the user. It is also to be noted that "r" will have all $\mathrm{k}$ users data and each spreading code assigned to each user in the transmitter will be used again in the receiver to get the single signal element from " $\mathbf{r}$ ". The number of spreading codes 
are equal to number of users. But the OFDM symbol length is the size of the spreading code.

$r=X S+\eta=\left(R_{0}, R_{1}, \ldots, R_{L-1}\right)$

$X=H C$

$X$ is called as system matrix.

\section{OPTIMUM RECEIVER}

The detector for the MC-CDMA can be of two types, namely Single-User detector and Multi-User detector. The Single-User detector is usually an suboptimal detector having an equalizer and quantizer combination.

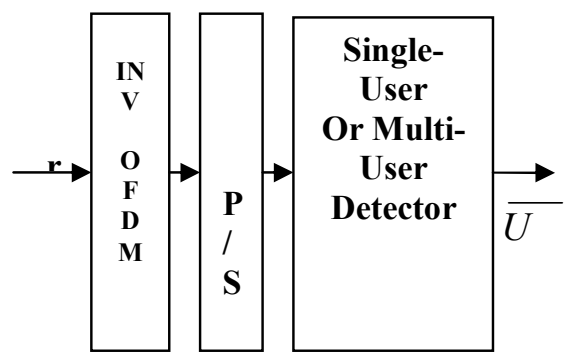

Figure 2. Receiver configuration of a MC-CDMA system

The equalizer of Single Tap zero forcing for example will lead to

$\overline{U^{\prime}}=E r$

Where

$E_{l, l}=\frac{H_{l, l}^{*}}{\left|H_{l, l}\right|^{2}}$

$\bar{U}=q u a n t i z e\left(\overline{U^{\prime}}\right)$

The Multi-User detection can be of optimal or suboptimal [7]. In this research optimal detectors are considered and MLSE version of the optimal detector is formulated as below,

In MLSE, the received symbol is compared with all possible symbols from all users. The comparison is a Euclidean metric and the symbol which gives minimum value is considered as the transmitted one. For a symbol of size $L$ and users $K$, the number of Euclidean distance calculation is $L^{K}$.

$$
E_{s e}=\left\|r-X U_{L}\right\|^{2}
$$

The optimal detectors are mandatory when there is a severe MAI due to the fading channel characteristics and clipping noise. The clipping noise effects has been already studied in detail in $[10,11]$ and models to generate clipping noise effects has also been proposed. The performance of MCCDMA system under such impairments is discussed in detail and BER performance analysis for a Zero Forcing equalizer aided detectors are shown [12].

\section{CLIPPING NOISE EFFECTS}

The OFDM uses High Power Amplifiers(HPA) which are usually non linear for achieving high efficiency. This amplifiers however does not have equal gain throughout the frequency range. This leads to clipping of few symbols and usually called as clipping noise. This makes OFDM to suffer from spectral spreading and In-Band distortion. This clipping noise reduces spectral efficiency and BER performance. One of the solution is using Golay Sequences for small number of users and Walsh Hadamard sequences for high number of users.

The modeling of the clipping effect on the OFDM signal is shown in $[10,11]$ for a distorting system with characteristics denoted as $f(x)$. Let $x(k)$ be the discrete OFDM signal in time domain which follows the complex Normal distribution with equal variance $\mathrm{Px} / 2$.. When this signal is passed through a clipping amplifier, the resultant will be of two components. One component will be the signal itself and the other is the distorted component. This can be easily verified by the Bussgang Theorem and shown in[10].

$$
X_{c l i p}(k)=\alpha X(k)+n_{d}(k)
$$

Alpha depends on the $P_{x}$ and $f(x)$. The clipped signal is

$$
\begin{aligned}
& X_{\text {clip }}(k)=\{X(k)\} \text { when }|X(k)<A| \\
& X_{\text {clip }}(k)=A^{\arg (X(k))} \text { when }|X(k) \geq A|
\end{aligned}
$$

The Input power BackOff (IBO) for such systems is given by

$$
I B O=\frac{A^{2}}{P_{x}}
$$

This IBO is given usually in decibels and variation of the IBO changes the performance analysis in terms of BER $[10,11]$.

\section{SPHERE DECODING}

The principle of the algorithm is to search the closest lattice point to the received signal within a sphere of radius $\sqrt{ } \mathrm{C}$ centered at the received signal. The choice of $\mathrm{C}$ is very crucial to the speed of the algorithm. In practice, $\mathrm{C}$ can be adjusted according to the noise (and eventually the fading) variance. When a failure is detected, one can either declare an erasure on the detected symbol, or increase C .

The complexity of the algorithm is independent of the lattice constellation size, which is very useful for high data rate transmission. 


\section{GLOBAL SEARCH AS AN OPTIMAL DETECTOR}

In Section 3, it is clearly shown that MC-CDMA system performance is poor when there is a non linear distortion like clipping noise or when the channel characteristic are time dispersive. Under such situation optimum detection using Exhaustive Search method is only solution. However Exhaustive Search method is computationally expensive and alternatives like Sphere Decoding and Genetic Algorithms are proposed [6,7]. Another approach of using Particle Swarm Optimization is also proposed to assist the Multi User Detection (MUD) procedure [13]. In this paper a new global optimization technique called Global Search (GS) algorithm is used [9].

The main idea behind Global Search(GS) algorithm is to find find starting points for gradient based local NLP solvers. OptQuest is a scatter search developed by OptTek ${ }^{\circledR}$ Systems is used to find the starting point for such gradient search algorithm. The combination of global optimization along with powerful local search makes this algorithm very effective. In this reference it has been proved that this algorithm is capable of solving complex optimization problem with linear and non linear constraints easily. The Scatter Search is an augmentation of GA where new population is generated in a deterministic way rather than the random way.

\section{SIMULATION RESULT}

The MC-CDMA system as proposed in [6] is taken into study in this paper. This serves as a tool to compare the performance analysis of GS algorithm when compared to the available optimal detectors.

The scenario similar to reference [6] is taken into consideration in this part of analysis. The received symbol power is equal to the transmitted symbol power. Each user symbol is spread over $L=64$ sub-carriers, 32 users with a real Walsh-Hadamard sequence and the guard interval is $25 \%$ of the OFDM symbol period employing QPSK is taken into consideration. The system is simulated in MATLAB ${ }^{\circledR}$ environment and $10^{8}$ symbols are transmitted and received.

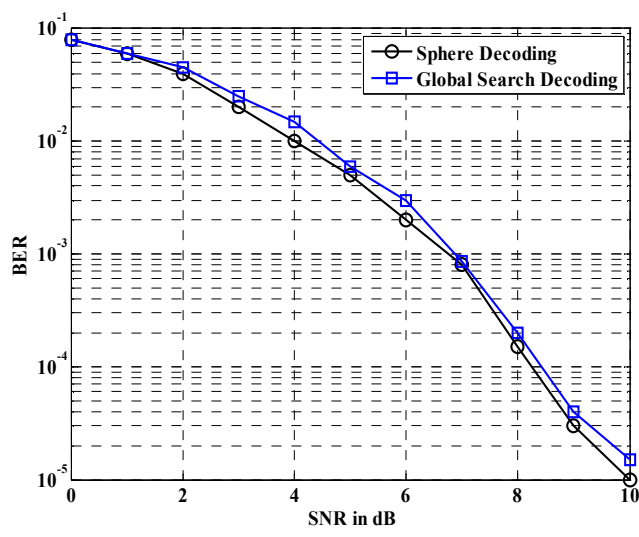

Figure 3. BER performance of Global Search Algorithm when compared with Sphere decoding algorithm

In Figure 3, the comparison between the Sphere Decoding algorithm and GS algorithm is done. The performance of GS algorithm is comparable with the Sphere Decoding algorithm based MUD.

\section{CONCLUSION}

In this paper the Global Search algorithm is used to assist Multi User Detection in MC-CDMA affected clipping noise. The simulations are done with respect to literature found. It has been clearly shown that the Global Search algorithm is a suitable candidate for the MUD problem compared to the Sphere decoding. The main limitation is lack of tuning the algorithm and computational complexity comparison between existing algorithms. However the parallel processing capability of the GS algorithm is definitely a positive signal to use this technology in the detection.

\section{REFERENCES}

[1] S. Hara and R.Prasad, "Overview of Multicarrier CDMA" IEEE communication magazine, Dec 1997, pp 126-133.

[2] N. Yee, J.-P. Linnartz, and G. Fettweis, "Multi-carrier CDMA in indoor wireless radio networks," in Proc. IEEE PIMRC'93, Sept. 1993, pp.109-113.

[3] K. Fazel and L. Papke, "On the performance of convolutionally-coded CDMA/OFDM for mobile communication system," in Proc. IEEE PIMRC'93, Sept. 1993, pp. 468-472.

[4] Shinsuke Hara, and Ramjee Prasad, "Design and Performance of Multicarrier CDMA System in FrequencySelective Rayleigh Fading Channels" IEEE Transactions On Vehicular Technology, Vol. 48, No. 5, September 1999 pp 1584-1596

[5] Ebrahim Soujeri, Huseyin Bilgekul "Hopfield Multiuser Detection of Asynchronous MC-CDMA Signals in Multipath Fading Channels" IEEE Communications Letter, Vol. 6, No. 4, April 2002. pp147-156.

[6] Loïc Brunel "Optimum Multiuser Detection for MCCDMA Systems Using Sphere Decoding" in Proc IEEE International symposium on PIMRC,San Diego.California. Vol 1,pp A16-A20,Oct 2001

[7] Hua Wei and Lajos Hanzo "Genetic Algorithm Assisted Multiuser Detection for Asynchronous Multicarrier CDMA" IEEE VTC'04 (Fall), 26-29 September 2004, Los Angeles, USA. pp. 1914-1918.

[8] Qinghua Shi, Yong Liang Guan, Yi Gong, and Choi Look Law, "Receiver Design for Multicarrier CDMA Using Frequency-Domain Oversampling" IEEE Transactions On Wireless Communications, Vol. 8, No. 5, May 2009 pp 2236-2241

[9] Ugray, Zsolt, Leon Lasdon, John C. Plummer, Fred Glover, James Kelly, and Rafael Martí. "Scatter Search and Local NLP Solvers:A Multistart Framework for Global Optimization" INFORMS Journal on Computing, Vol. 19, No. 3, 2007, pp. 328-340.

[10] X. Li and L. J. Cimini Jr., "Effects of clipping and filtering on the performance of OFDM," IEEE Communications Letters, vol. 2, no. 5, pp. 131-133, 1998.

[11] Hideki Ochiai, Hideki Imai"Performance of OFDMCDMA with Simple Peak Power Reduction" European Transactions on Telecommunications, Volume 10 Issue 4, Pages $391-398$ 
[12] S.Sivanesskumar and R.Sukanesh " Performance analysis of Miulti-Carrier Code Division Multiple Access system under Clipping noise" European Journal of Scientific Research, Vol. 38 No. 4 pp590-595

[13] Hung.H.L., and Wen.J.H., "An Adaptive Multistage Multiuser Detector for MC-CDMA communication
Systems Using Evolutionary Computation Tecnhique" Wireless personal communication Journal, Springer 2009.

[14] H. Ochiai and H. Imai, "Performance analysis of deliberately clipped OFDM signals," IEEE Transactions on Communications, vol. 50, no. 1, pp. 89-101, 2002. 